\title{
Coulisses
}

Revue de théâtre

13 | Hiver 1996

Varia

\section{Peepshow dans les Alpes de Markus Köbeli}

\section{Christine Friedel}

\section{(2) OpenEdition}

Journals

Édition électronique

URL : http://journals.openedition.org/coulisses/4315

DOI : $10.4000 /$ coulisses.4315

ISSN : 2546-9460

\section{Éditeur}

Presses universitaires de Franche-Comté

\section{Édition imprimée}

Date de publication : 1 janvier 1996

Pagination : 70-72

ISSN : 1150-594X

\section{Référence électronique}

Christine Friedel, «Peepshow dans les Alpes de Markus Köbeli », Coulisses [En ligne], 13 | Hiver 1996,

mis en ligne le 15 mars 2019, consulté le 05 octobre 2020. URL : http://journals.openedition.org/ coulisses/4315 ; DOI : https://doi.org/10.4000/coulisses.4315

Ce document a été généré automatiquement le 5 octobre 2020.

Coulisses 


\title{
Peepshow dans les Alpes de Markus Köbeli
}

\author{
Christine Friedel
}

Création française à Besançon, au Nouveau Théâtre-CDN, du 3 au 18 novembre 1995.

Traduction : Jean Launay. Mise en scène : René Loyon. Décor : Nicolas Sire. Costumes : Françoise Luro. Son : Emmanuel Mathey. Avec: Jean-François Lapalus, Chantal Mutel, Muriel Racine, Nicolas Dufour, Claude Monnoyeur.

1 Une idée reçue en voie de révision : la comédie serait aujourd'hui la chasse gardée d'un théâtre de simple divertissement, au sens strict du terme : ce qui détourne le public de ses inquiétudes sur le monde, et, plus profondément, de l'angoisse de la condition humaine. Il est vrai qu'une écriture dramatique soucieuse de rendre compte du monde d'aujourd'hui endosse le plus souvent le pessimisme ambiant - «Y en aurait, des choses à dire » (réplique coupée d'un spectacle passé) -, grevée de surcroît par l'esprit de sérieux.

2 D'autre part, la part du pauvre accordée à l'écriture contemporaine par les institutions théâtrales et par le public, qui se méfie de textes sans références, les difficultés de productions conduisant à une autocensure (écrire des monologues ou des pièces exigeant peu d'acteurs), la solitude des auteurs qui ne travaillent pas toujours avec des acteurs, des praticiens de la scène, n'incite pas à l'écriture de comédies. En réaction à ces phénomènes, un certain nombre d'actions volontaristes, dont la plus spectaculaire, inventée par le Centre National des écritures du Spectacle, à la Chartreuse de Villeneuve-les-Avignon : la « nuit de la comédie », en décembre 1991, où des acteurs ont lu, de cinq heures du soir à cinq heures du matin, les pièces dont commande avait été passée à sept auteurs (Catherine Bidaut, Sylvie Chenus, Olivier Dutaillis, Alain Gautré, Jean-Yves Picq, Anita Van Belle).

3 L'humour revient au théâtre qui se veut citoyen et éveilleur, avec des auteurs comme Noëlle Renaude, ou avec la première comédie d'Eugène Durif. Reste que la critique 
nationale rend actuellement avec éclat hommage aux grands comiques solitaires (célébration de Valérie Lemercier dans Le Monde et au Masque et la Plume), rois d'un genre particulier : la satire de "types sociaux », jouant sur un effet de reconnaissance immédiat, très efficace, et qui ferme le plus souvent le débat. On rit de l'Autre, et ça détend.

Peepshow dans les Alpes est bien au cœur du débat sur les limites des genres: où est la caricature, où est la satire? Il s'agit de la seconde pièce - la première, Zimmer frei, Chambre libre, n'est pas encore traduite en français-de Markus Köbeli, auteur de satires pour la radio et la télévision suisse alémanique. Voilà un homme qui a tous les moyens, largement diffusés, de s'exprimer. Pourquoi le théâtre? Parce qu'il sait bien que l'écran et les ondes unifient, arrondissent le propos, quoi qu'on puisse dire, et aussi délectables et féroces que soient certaines émissions satiriques. La présence des acteurs vivants confère évidemment au propos de l'auteur un trouble, un poids de réalité qui peut être poignant au cœur même du rire.

5 L'acteur vivant - et plus encore les liens difficilement explicables qui se tissent dans une « distribution»"- a pour spécialité de mettre les doigts, et le reste, dans la fissure involontaire (surtout dans un texte aussi bien «bouclé» que Peepshow), dans l'inconscient du texte. C'est peut-être là la différence avec le «one man, ou woman, show » : à la différence du texte sur mesure, écrit pas l'interprète lui-même, l'écart jamais totalement réduit entre les acteurs et l'écriture.

6 L'important étant de ne pas se tromper de rire. Peepshow dans les Alpes - titre provocateur s'il en est - n'est pas une gaudriole sur les péquenots, bouseux et autres ploucs. Ce qui est visé, c'est la mise en spectacle «fatale »-diraient les tenants du "marché"-, et donc vulgaire, d'un mode de vie en déclin. En choisissant la forme spectaculaire la plus misérable - il suffit de voir, par les rues chaudes des grandes villes, les réclames pour les « live-show » à prix cassés -, Markus Köbeli pointe la dégradation de la représentation dans une société qui veut aller trop vite à l'argent, instantanément, sans réel investissement.

7 Qu'on en juge par le résumé de la pièce: la famille Holzer ne peut plus vivre de l'agriculture. La petite station de sport d'hiver où elle vit est supplantée par les grandes, en haute altitude. Or le fils, Hans junior, et un entrepreneur de cars avisé, ont remarqué qu'en montant plus haut les touristes s'arrêtent pour se soulager et regarder par la fenêtre. L'idée vient très vite de les faire payer pour cela.

Hans (le père) :

Oui.

Ils veulent voir

comment ce serait

si c'était

comme c'est encore maintenant.

C'est comme ça. 
Peepshow dans les Alpes

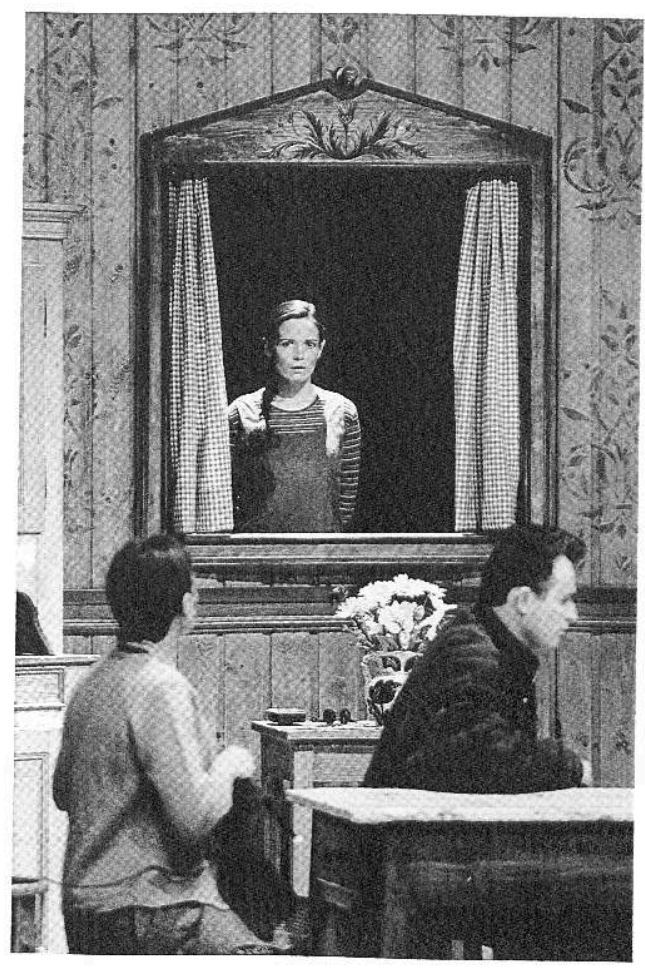

Mais être authentiquement soi-même quand on est regardé derrière une vitre payante est bien difficile. La famille se trouvera alors dans la situation d'un groupe de théâtre amateur qui cherche une pièce, voit «ce qui marche » et répond à la « demande » en sacrifiant précisément, au nom de 1'«authenticité » demandée par un public virtuel, toute authenticité: Heidi en costumes de poupées Barbie, portant à son comble l'inauthentique, marquera pour les Holzer la fin de l'aventure. Ils y auront gagné de l'argent, le désœuvrement et l'éclatement de la famille, ils seront entrés dans la " société du spectacle ", sans racines et sans perspective.

La satire a besoin de grands écarts : l'écart entre le mode de vie traditionnel qui était celui des Holzer au début de la pièce et le monde des "parcs d'attraction"; et de la condensation du temps : à chaque acte $(2,3$ et 4$)$, une tentative de "spectacle » est épuisée, en cinq actes et une année, tout est joué. Ce qui est joué, c'est l'irrésistible substitution au « commerce », y compris entre les hommes, du «marché »-y compris par l'usage du play-back, dans des langues à fortes devises et à gros potentiel touristique. Où l'on voit que le «spectacle» a besoin de s'approprier une parole univoque et porteuse de profit, et de faire taire ses sujets.

La satire est par nature démonstrative : elle fonctionne, dans l'écriture, comme une expérience scientifique; sur un terrain donné - ici, celui du naturalisme, avec la présentation à l'état "naturel » de la famille Holzer-, elle introduit une donnée nouvelle - ici, l'irruption de la forme spectaculaire dominante - et donne à constater les résultats, d'où l'on peut tirer une loi - ici, celle du circuit le plus court entre le spectacle et l'argent d'un côté, et de l'autre celle de la dislocation accélérée d'un système social sous la poussée du spectacle «marchandise »; le tout sans nostalgie. La satire est donc pédagogique : celle de Markus Köbeli est rigoureusement construite ; elle commence par la lecture et le commentaire du journal, et place ainsi d'entrée le 
spectateur dans le champ du médiatique, qui ne s'ouvrira aux personnages de la pièce qu'à mesure de la « progression » du spectacle qu'ils offrent.

11 Cette construction détermine fortement la mise en scène: le spectacle dans le spectacle, la logique du grand écart, induit un «fond » naturaliste, c'est à dire un code de jeu qui permet d'identifier immédiatement des personnages, leurs liens sociaux et affectifs, leur situation, en référence à un réel lui-même identifiable. Ce qui sauve ce naturalisme du péché de confusion et de substitution de l'image au réel - le sujet même de la pièce -, c'est sa mise en théâtre visible : ici, entre autres, par la présence d'un décor qui signifie le chalet tout en renvoyant, par sa structure tournante, au pur théâtre (signe du passage du temps, rôle de double voyeur donné au public, allusion au « tournez manèges » de la foire...). Autre exemple : peut-être aurait-il fallu donner une présence sensible au public massé derrière les fenêtres (silhouettes, bande-son...) ? Son absence, en tout cas, le situe radicalement où il est, public virtuel qu'on n'a pas plus besoin d'identifier que "la ménagère de moins de cinquante ans" visée par les audimat ; c'est précisément son caractère virtuel qui constitue l'un des enjeux de la pièce.

12 Un dernier exemple: le personnage du grand-père: intégré aux séquences «naturalistes» de la pièce, il est cependant un personnage "masqué », une marionnette humaine. Il s'agit là d'un choix de mise en scène, de l'utilisation esthétique des contraintes de la troupe : avec ce jeu masqué d'un jeune homme donnant à voir un très vieil homme, on est déjà dans le théâtre du théâtre, et dans le sujet de la pièce, un monde de symboles vivants (vivant à peine, dans ce cas...).

La forme satirique - texte et mise en scène - de Peepshow dans les Alpes a un mérite : elle est immédiatement partageable par le public populaire. Et un inconvénient: elle ne souligne pas sa propre modernité, à l'intention d'un public de connaisseurs préoccupé $\mathrm{du}$ « comment »- bonne question - mais aux dépens et non au nom du «quoi ». Or ce formalisme rejoint, dans ses symptômes, la question même développée dans la pièce : quelle est la forme qui « paie »?

D'un côté, dans la fiction, le règne du show à l'américaine, concentré et perverti dans le "Peepshow», où le regard voyeur assume l'individualisme et donc la frauduleuse « authenticité » $d u$ «j'y étais ». De l'autre, en une période de - discutable - « mort des idéologies ", l'appel à une modernité réduite à l'immédiate affirmation d'une originalité, auto-contemplative au détriment d'une pensée partageable.

14 Markus Köbeli a choisi de résoudre la question formelle par la satire classique : cela lui a permis d'être entendu et joué (au-delà de la Suisse, la pièce a été jouée dans quarante théâtres en Allemagne, et quelques-uns en Angleterre et aux Pays-Bas).

Et René Loyon a mis la pièce en scène dans toute la rigueur - à laquelle on peut reprocher d'être trop bien «bouclée » - de sa construction, tout en ouvrant aux acteurs ce qu'elle laisse malgré elle d'espace. Dès lors, ça joue... 